\title{
PERFORMARE IL PERTURBANTE UNA PROPOSTA DI DEFINIZIONE DEL FANTASTICO TEATRALE CONTEMPORANEO A PARTIRE DALL'UNHEIMLICH DI FREUD
}

\author{
MONICA CRISTINI \\ Università degli Studi di Verona \\ mo.cristini@gmail.com \\ Recibido: 01-07-2014 \\ Aceptado: 14-11-2014 \\ (). (1)
}

SOMMARIO

Esiste un teatro di ricerca fantastico? Questo saggio nasce da una lettura del testo Das Unheimliche di Freud per articolarsi in un'analisi delle ultime argomentazioni proposte dalla critica letteraria. L'approccio pluridisciplinare rivolto agli studi teatrali, ma anche all'estetica e agli studi dedicati al perturbante emersi in ambito psicoanalitico, ha per obiettivo la costruzione di una base critica che sia utile riferimento per una riflessione sul teatro contemporaneo di ricerca italiano. Si propone uno studio inedito nel quale la ricerca sul fantastico si interseca con l'analisi semiologica degli elementi costitutivi del teatro postdrammatico, nell'intento di scoprire quali possano essere gli elementi costitutivi di un perturbante-fantastico teatrale.

PARole ChiAve: unheimlich, perturbante, fantastico, teatro di ricerca, postdrammatico, Sigmund Freud, Socìetas Raffaello Sanzio, Fanny \& Alexander, Teatro Valdoca

Abstract

Does a fantastic form exist in experimental theatre? This essay starts from the Freudian concept of Das Unheimliche to analyze the latest considerations suggested by literary critics. The argument derives from a multidisciplinary perspective including not only theatrical studies but also aesthetic ones and psychoanalytical research on the uncanny, aiming to build a critical base that will be a useful landmark for some preliminary considerations about Italian contemporary experimental theatre.

The essay therefore proposes an approach linking research about the uncanny with the semiological analysis of the constitutive elements of postdramatic theatre, 
with the aim of defining what the constitutive elements of a theatrical uncanny-fantastic might be.

KEYWORDS: Unheimliche, uncanny, fantastic, experimental theatre, postdramatic theatre, Italian contemporary theatre, Sigmund Freud, Socìetas Raffaello Sanzio, Fanny \& Alexander, Teatro Valdoca

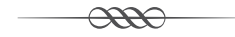

La ricerca sul fantastico sorta nel secolo scorso, e in principio orientata per lo più verso l'ambito letterario, ha successivamente volto l'interesse alle diverse forme dello spettacolo rilevando, nell'elaborazione di una definizione del fantastico stesso, nuove questioni epistemologiche. Come ben sottolinea Nicola Pasqualicchio nell'introduzione de La meraviglia e la paura (2013), ${ }^{1}$ solo negli ultimi anni gli studiosi hanno incominciato ad indagare il fantastico nel teatro e a tentare di rintracciarne le forme, sia a livello drammaturgico che nella realizzazione scenica, evidenziando non poche problematiche teoriche e metodologiche, non da ultimi il peculiare plurilinguismo che caratterizza le arti sceniche e la totale assenza di una pregressa letteratura critica. Se infatti da un lato può essere più semplice l'identificazione di un testo drammaturgico fantastico (o la presenza di elementi fantastici nel dramma), ${ }^{2}$ dall'altro si riscontra una maggiore difficoltà nel momento in cui ci si accinge alla stessa indagine rivolta verso la realizzazione scenica dell'opera. Ulteriori problematiche si evidenziano in una definizione del fantastico nel teatro contemporaneo. Se infatti, nonostante i limiti tecnici dell'epoca, può essere più immediata la dichiarazione di appartenenza alla forma fantastica di una rappresentazione

1 Volume che raccoglie gli atti del convegno di studi dedicato a teatro e fantastico, tenutosi a Verona nel 2011. Il convegno è stato curato dal gruppo di ricerca Teatro e Spettacolo Fantastico di cui faccio parte, formatosi nel 2009 presso il Dipartimento di Filologia Letteratura e Linguistica dell'Università di Verona e diretto da Nicola Pasqualicchio. Sin dall'inizio della ricerca l'oggetto di studio ha evidenziato la problematica di valutare l'effettiva esistenza di un «teatro fantastico», di definire il fantastico in teatro e nello spettacolo e delimitarne i confini. Si è scelto dunque di affrontare il lavoro a partire da un'analisi della critica letteraria rivolta al fantastico, individuando in seguito valenze e modalità rappresentative del fantastico nel teatro europeo a partire dal diciottesimo secolo.

2 Procedendo con un'analisi che si articoli a partire dalle definizioni elaborate dalla critica letteraria che ci indica gli eventuali elementi di appartenenza: la presenza di spiriti ed esseri soprannaturali, di vampiri, mostri, lupi mannari, oggetti che si animano, eventi straordinari e perturbanti, l'irrompere di elementi estranei alla realtà del racconto che mettono in crisi il giudizio critico del lettore, ecc. 
teatrale, operistica, o di un balletto del diciottesimo o diciannovesimo secolo, ${ }^{3}$ più complesso risulta rintracciare tale genere o forma nella drammaturgia contemporanea e soprattutto nel teatro di ricerca di questi ultimi anni.

Questo scritto nasce dunque da una riflessione su tale questione, a partire dal tentativo di dare una risposta alla domanda «esiste un teatro fantastico del nuovo millennio?» - con uno sguardo rivolto in particolare alla ricerca italiana degli ultimi vent'anni e agli elementi e situazioni perturbanti che talvolta emergono sulla nostra scena contemporanea. Come base critica, insieme agli studi sulla letteratura fantastica, ho assunto quale punto di partenza il saggio di Freud Das Unheimliche, ormai preso in considerazione da ogni studioso che si dedichi al fantastico, per poi estendere la ricerca alle riflessioni sul perturbante nella letteratura del Novecento e contemporanea e alle considerazioni apparse di recente nell'ambito degli studi sulla psicoanalisi. Infine, avendo scelto un approccio semiologico e iconografico, mi sono avvalsa di alcuni studi emersi in ambito estetico e nell'area delle scienze cognitive per chiarire la valenza del perturbante nel «teatro dopo l'età d'oro» (De Marinis, 2013): un teatro che segna l'azzeramento della tradizione del Novecento, centrata sulla soggettività espressiva dell'attore e che evidenzia invece «la costruzione di un modo di pensare e di fare teatro fondato su riferimenti differenti e in gran parte non teatrali, ma piuttosto artistico-visivi, fotografici, filmici, coreici, filosofici, etc.» (De Marinis, 2013: 16) - logica evoluzione di quella «prospettiva postdrammatica» che ha distinto il Novecento. È un teatro, quello contemporaneo, che porta i segni del superamento della rappresentazione (De Marinis, 2010); un fenomeno, come spiega De Marinis, con il quale la composizione scenica si è ri-creata su nuovi principi non più narrativi o illustrativi, mettendo in crisi le unità diegetiche della rappresentazione teatrale stessa; ${ }^{4}$ un teatro che, a mio avviso, rinunciando alla finzione paradossalmente si avvicina sempre più al fantastico: è questa la tesi che sono qui a proporre.

\section{DAS UNHEIMLICHE}

Il perturbante rientra in un genere di spavento che si riferisce a cose da lungo tempo conosciute e familiari.

$[\ldots]$

3 Sono numerosi in quegli anni numeri sensazionali con l'uso di botole o altri stratagemmi scenotecnici per rappresentare apparizioni e sparizioni. Cfr. La meraviglia e la paura, cit.

4 Nel fondamentale testo di riferimento Postdramatisches Theater (1999), Hans-Thies Lehmann evidenzia i dispositivi postdrammatici della frammentazione, la discontinuità, la simultaneità, la sospensione del senso e l'opacizzazione dei segni, inclinazione al paradosso e al disorientamento. 
Secondo lui [Jentsch] il fattore essenziale per l'insorgenza della sensazione di perturbamento risiede nell'incertezza intellettuale, per cui il perturbamento in effetti sarebbe qualcosa in cui non si sa come raccapezzarsi.

[...]

Però rileviamo che Schelling dice una cosa che illumina il concetto di unheimlich, in un modo inaspettato. Secondo lui è unheimlich tutto ciò che doveva rimanere segreto ma è venuto alla luce. (Freud, 2006: 222-226)

Nel saggio apparso su Imago nel 1919 Freud analizza varie ipotesi di definizione dell'unheimlich sia nella realtà che in letteratura, spiegando come esso sia identificativo di qualcosa che si avvicina a ciò che è spaventoso e che suscita orrore. In breve, ripercorrendo le definizioni date da Schelling, Jentsch e Rank, e avvalendosi anche dell'esperienza di alcuni casi clinici, il padre della psicoanalisi delinea alcuni fattori scatenanti la trasformazione di un fatto pauroso in un fatto perturbante: l'animismo, la magia, la stregoneria, l'onnipotenza del pensiero, la ripetizione involontaria, il complesso di castrazione, la follia. Nella narrazione letteraria alcuni elementi esemplificati sono invece il dubbio che un essere inanimato sia veramente vivo (figure di cera, pupazzi, automi, ma vanno aggiunte anche le manifestazioni di pazzia e gli attacchi epilettici poiché inducono nello spettatore l'impressione di processi automatici e fuori controllo); il fenomeno del doppio; ${ }^{5}$ la continua ricorrenza della stessa cosa (coazione a ripetere, il cui carattere perturbante è dovuto alla percezione di un non-dominio di sé); la visione di membra separate dal corpo (soprattutto se dotate di attività autonoma); silenzio, oscurità e solitudine.

Vorrei ora sottolineare alcuni punti del ragionamento di Freud che ritengo rilevanti per questa analisi e ai quali farò in seguito riferimento, poiché ciò che mi preme comprendere è l'insorgere della sensazione unheimlich nello spettatore. Indubbiamente non tutto ciò che è perturbante è anche fantastico; è di fatto assodato che a teatro una scena di violenza o un'azione inconsueta per lo spettatore siano unheimlich pur non contenendo elementi che possano essere considerati fantastici. Ma perché viviamo come perturbante la deformazione o manipolazione di un corpo, la presenza in scena di personaggi non ben riconoscibili, l'apparente ibridazione uomo-animale, un montaggio privo di narrazione? Credo allora che una riflessione sulle argomentazioni proposte da Freud possa essere d'aiuto per una nuova formulazione del fantasti-

5 In particolare Otto Rank (2001), nel suo saggio dedicato al doppio e pubblicato su Imago nel 1914, spiega come il doppio sia collegato alla paura della morte intesa come «allontanamento dall'io»: ciò che è intollerabile è perciò la paura di perdere se stessi. Di qui si deduce anche il terrore degli specchi o dei propri ritratti, strumenti ai quali viene attribuito il potere di «rubare» l'identità. 
co-perturbante nel teatro contemporaneo. Ecco dunque quelli che ritengo i punti focali del suo discorso.

1. Pur mettendo in dubbio la definizione di Jentsch, Freud nel corso del suo ragionamento sembra ricredersi e confermare infine l'incertezza intellettuale come uno dei principali fattori che contribuiscono all'insorgere del sentimento unheimlich. Ciò che sembra accomunare le esperienze vissute e quelle esperite dal lettore nella lettura è il fatto che un effetto di questo tipo si manifesta nel momento in cui viene meno la discriminazione tra immaginazione e realtà. Quando nella realtà si presenta qualcosa che fino a quel momento si riteneva immaginario o ad essa non appartenente, allora si manifesta nel lettore un sentimento perturbante. Lo spaesamento insorge dunque nel momento in cui non si riconosce più la realtà in cui ci si trova, che sia essa vissuta o rappresentata.

2. Freud chiama in causa anche Schelling, spiegando che «può essere vero che il perturbante sia un fatto che, nell'intimo, è familiare, e riemerge dopo essere stato sottoposto a rimozione» (2006: 243). Un'esperienza rimossa, dunque, che si riaffaccia di fronte ad un'immagine o a un avvenimento che la richiama. Unheimlich si riferisce così a cose conosciute e familiari (heimlich) che in alcune occasioni diventano inquietanti. ${ }^{6}$

3. Il fatto che, nonostante alcune credenze siano state superate, l'uomo contemporaneo si ritrovi, di fronte a determinati eventi, a dubitare delle nuove: di qui l'insorgere del sentimento unheimlich. Freud spiega come, nonostante siano state superate, le nostre vecchie credenze o quelle appartenenti ai nostri progenitori continuino a vivere in noi a livello inconscio e tornino alla luce, riconfermate, ogni qualvolta un evento le possa richiamare: è proprio questa dualità che crea il senso di perturbamento.

La situazione si trasforma non appena lo scrittore finge di trasferirsi nel mondo della realtà corrente. In questo caso egli accetta anche tutte quelle condizioni che contribuiscono al manifestarsi di sensazioni di perturbamento nella vita reale, e tutto quello che avrebbe un'efficacia perturbante nella realtà lo avrà anche nella narrazione, ma, adesso, lo scrittore è addirittura costretto ad accrescere e moltiplicare gli effetti, molto oltre le possibilità del reale, descrivendo eventi che di fatto non accadono mai o sono estremamente rari. Con questo egli ci getta, in un certo senso, nelle braccia di quella superstizione che, apparentemente, avevamo superato; ci inganna promettendo di darci la nuda verità, mentre poi la trasgredisce. Noi reagiamo alle sue fantasie allo stesso modo in cui

6 Come spiega Graziella Berto (1999), «la negazione un- non cancella, ma svela; e svela il segreto dello Heim, della dimora, del proprio: il suo mantenersi, dimenticato, nello Heimliche in quanto nascondimento [...]. La dimensione del vicino, del proprio, non è così tranquillizzante e controllabile come normalmente si ritiene: questo è il segreto che nell'Unheimliche viene alla luce» (1999: 24-25). 
reagiremmo ad esperienze reali. Quando, alla fine, scopriamo il trucco, ormai è troppo tardi e l'autore ha conseguito il suo scopo. (Freud, 2006: 248)

4. Vorrei infine sottolineare, dopo questa introduzione al saggio di Freud, come egli giunga a ritenere che debba essere fatta una distinzione tra il perturbante vissuto e il perturbante immaginato o letto.

Effettivamente, il perturbante, quale è rappresentato nella letteratura, nei racconti e nelle opere di fantasia, merita di essere considerato a parte. Il suo dominio, soprattutto, è di gran lunga più ricco di quello del perturbante nella vita reale, dato che contiene integralmente quest'ultimo e, inoltre, altre cose ancora, che non si ritrovano nella vita vera. Non è possibile trasferire, senza profonde modificazioni, al dominio del perturbante letterario il contrasto tra quel che è stato rimosso e quel che è stato superato. Infatti il regno della fantasia, per realizzare i propri effetti, deve fondarsi sull'impossibilità della verifica. Ne deriva una conseguenza piuttosto paradossale, cioè che, innanzi tutto, molto di ciò che non è perturbante nell'opera di fantasia, lo sarebbe se si attuasse nella vita e, in secondo luogo, la letteratura fantastica dispone di molti più mezzi, che non la vita, per creare effetti perturbanti.

Lo scrittore di narrazioni fantastiche gode, tra molte altre libertà, della facoltà di scegliere il proprio mondo di rappresentazioni, così da riprodurre la realtà consueta o da discostarsene come meglio gli piaccia. (Freud, 2006: 247)

Mentre l'influenza dell'ambiente che ci circonda ci porta ad assumere un atteggiamento passivo nei confronti dell'esperienza reale, il narratore può esercitare sul suo pubblico un'azione diretta e stimolare nel lettore l'insorgere di determinate emozioni.

Il perturbante può essere dunque definito un fenomeno che sorge dalla rappresentazione (letteraria) e dall'identificazione nella lettura; si potrebbe dire che è la realtà oggettiva della fantasia inconscia del lettore che torna alla coscienza. Come spiega Adam Bresnick (2001), dal punto di vista psicoanalitico un'opera artistica produce effetti reali nel pubblico ed è dunque qualcosa che si esperisce: anche Freud, infatti, attribuisce all'opera artistica un effetto estetico grazie alla sua funzione catartica. D'accordo con alcune formulazioni di Freud, Bresnick definisce il perturbante come un momento di straniamento che arriva al ritorno di una memoria da lungo repressa, «a revenant mnemonic trace that occupies a kind of interior no-man's land, belonging neither to the conscious nor to the unconscious, but to both at once» (2001: 117).

Credo che a questo punto sia essenziale introdurre un'altra teoria, quella junghiana, ovvero che le immagini che tornano alla memoria non ap- 
partengano soltanto alla soggettiva esperienza dell'individuo ma siano frutto anche di una memoria collettiva e per questo definibili archetipi appartenenti ad un immaginario comune e universale. Ipotesi che giustificherebbe l'insorgere del perturbante di fronte a figure o fatti che non riconosciamo e sembrano non appartenere alla nostra memoria personale. Come spiega Jung (2004), queste «immagini primordiali» che crescono nell'inconscio dell'uomo moderno non possiedono nessun carattere soggettivo, ma sono del tutto collettive e riaffiorano in virtù di una naturale funzione psichica.

Si tratta della reviviscenza di un archètipo, [...]. È il modo di pensare analogico e primitivo del sogno, che ricostruisce queste antiche immagini. Non si tratta di rappresentazioni ereditarie, ma di facilitazioni ereditarie.

In considerazione di questi fatti, dobbiamo dunque ammettere che l'inconscio contenga non soltanto elementi personali, ma anche elementi impersonali, collettivi, in forma di categorie ereditarie, o archetipi. (Jung, 2004: 42)

\section{ELEMENTI PER UNA NUOVA DEFINIZIONE}

I saggi dedicati al fantastico - a partire da Todorov (2007 [1970]), Ceserani (1996) e Caillois (1984 [1965]) - sembrano concordare nell'attribuire al racconto il potere di suscitare nel lettore l'insorgere di una sensazione inquietante e perturbante e derivante da un evento che trasgredisce le regole della realtà in cui si dispiega. Dalla stessa critica letteraria sono stati individuati alcuni elementi che concorrono a questa trasgressione e che però - va sottolineato sono stati scelti al fine di avvallare una definizione di fantastico formulata su un limitato campione di esempi: l'animarsi di oggetti inanimati o artificiali; l'apparizione di spiriti o il ritorno in vita dei morti; l'ibridazione tra uomo e animale e tutte le possibili deformazioni del corpo; il doppio (da cui la paura per gli specchi o i ritratti) e del sosia; la ripetizione dello stesso avvenimento o il reiterarsi di un'azione. Elementi, questi, che se inseriti in una dimensione che loro non appartiene, provocano nel lettore-spettatore un'esitazione interpretativa derivante dal problema a riconoscere la realtà in cui si trova: «[...] la definizione generale che se ne può trarre indica il fantastico come l'irruzione nel mondo reale di fenomeni inspiegabili sulla base di un'interpretazione razionale della realtà, con la conseguenza di creare nel protagonista e nel lettore con esso identificantesi una sensazione di sconcerto e un'incertezza interpretativa sull'origine e la natura degli eventi» (Pasqualicchio, 2013a: 10).

Negli ultimi anni la nuova critica letteraria ha rivolto l'interesse al fantastico contemporaneo, incentrando i propri studi su racconti e romanzi del No- 
vecento nei quali sembra dominare sempre più la valenza perturbante, o destabilizzante, di alcuni degli elementi riconosciuti dalla precedente critica, ma che ora si fanno specchio delle ansie e delle paure dell'uomo contemporaneo. Il fantastico sembra essere sempre più espressione e figurazione di una società in crisi e di un'umanità che riflette sul proprio valore e sulla propria identità, incarnando nell'elemento perturbante le inquietudini di un'intera generazione. Come ben ricorda Paola Di Natale (1995), come le altre forme di scrittura, quella fantastica è un'interpretazione della realtà. La studiosa spiega che, anche nel dare corpo all'impossibile - all'oltre-reale, nel trasformare in immagini ciò che è intuito - si agisce direttamente a partire da una interpretazione soggettiva e personale e di conseguenza l'opera ne tradirà necessariamente la sua matrice originaria, il materiale da cui è stata generata. Infatti, anche quando irrompe sulla scena, l'elemento fantastico si presenta con dei caratteri riconoscibili al lettore-spettatore e l'effetto perturbante è dato proprio dalla trasgressione e dalla deformazione di elementi che appartengono alla «normale esperienza fenomenologica». Si è dunque in presenza della «riconoscibilità del'inverosimile e l'irriconoscibilità del verosimile: il «mostruoso» - l'impossibile, l'insolito, l'irreale - è costruito a partire da elementi familiari e consueti, diversamente combinati o evocati per negazione, sì da sortire un effetto perturbante, ma contemporaneamente esso proietta un'ombra destabilizzante sul familiare e sul consueto, al punto da renderlo irriconoscibile ed estraneo» (Di Natale, 1995). ${ }^{7}$

A quanto pare, il fantastico non può fare a meno di valersi di forme che si possano riconoscere o di immagini-archetipo che appartengano alla memoria collettiva e che si combinino a paure o a conflitti rimossi e latenti.

Gli esseri mostruosi, di cui sono popolati il mito e letteratura fantastica [...] traducono dunque in maniera paradigmatica la tensione dialettica tra partecipazione e distanziamento, tra il visibile e il possibile, tra ciò che è considerato normale e ciò che viene percepito come alieno; ipostatizzando, nella forma dell'ibridazione, il conflitto tra l'umano e l'extra-umano, tra la verità, approssimativa e di continuo suscettibile di aggiustamenti, quale si disvela all'esperienza, e l'interpretazione, necessariamente allusiva e ambigua. Che incarnino il prodigioso proiettato nell'outspace, la variabile di alterità percepita in uno spazio esterno al soggetto, oppure il mistero degli orizzonti interiori dell'inconscio, la deformità assorbita dal soggetto, essi manifestano in modo macroscopico la saldatura tra le dimensioni dell'essere e del conoscere.

7 La studiosa spiega che ogni atto interpretativo allo stesso tempo rimescola e modifica materiali preesistenti e produce invenzioni. Nell'atto creativo si sovrappongono dunque «delirio e conoscenza»; ciò spiega come le immaginazioni emergano dall'esperienza con un carattere di concretezza. 
[...] il mostro continua ad essere indicatore di un'eccedenza semantica, portatore di una significazione che scatena il dramma ermeneutico, segno che allude metaforicamente ad una conoscenza «altra»: del reale, vero labirinto inestricabile in cui l'inverosimile si nasconde nelle pieghe del quotidiano - rassicurante e perciò fallace -, e di se stessi. (Di Natale, 1995)

La studiosa chiarisce come il fantastico, superando i limiti dell'oggettività, infranga la realtà e porti lo spettatore a dubitare della propria interpretazione, sospendendo la fiducia «non tanto nel reale quanto del verosimile, poiché mette in discussione non i fatti ma le categorie con cui i fatti vengono interpretati» (Di Natale, 1995). La valenza cognitiva del fantastico risiede perciò nel creare nuove categorie d'interpretazione e di conseguenza di produzione di nuove realtà. È così che l'esperienza destabilizzante del fantastico dà vita a un'immagine «seducente del reale»:

[...] introduce una spinta contraria, dalla quale può prendere origine lo scatto critico, il barlume di una coscienza che simultaneamente aderisce alle pieghe del mondo e lo comprende all'interno da un lato e dall'altro si proietta al di fuori, ragionando su di esso. Ciò perché il fantastico è per eccellenza campo generatore di tensioni: tra partecipazione e distanziamento, identificazione e rigetto, su cui la sospensione getta un ponte percorribile ad ogni momento nelle due direzioni. (Di Natale, 1995)

La conclusione a cui arriva Di Natale sarà un utile spunto di riflessione nel momento in cui rivolgerò la mia analisi all'ambito teatrale. Infatti, nel teatro di ricerca gli elementi fantastici sembrano rispecchiare in modo sempre più decisivo le tensioni e le ansie di una società in cui l'individuo sembra perdersi alla ricerca della definizione di un sé non più immediatamente riconoscibile, portando sulla scena figure mitiche e proprie dell'immaginario fantastico-perturbante che inducono nello spettatore un disorientamento che conduce alla riflessione.

In altri studi dedicati al fantastico contemporaneo si evidenzia come esso non si identifichi più in realtà altre, lontane da quella della vita, e come si ancori invece a fatti veri, insinuandosi nella dimensione del quotidiano deformando il conosciuto, il familiare, e rendendolo ignoto e perturbante attraverso immagini straordinarie della nostra realtà. Il fantastico nel Novecento si fa allora riflessione sulla perdita d'armonia con se stessi e con il mondo e sulla minaccia derivante dalla presenza di eventi straordinari. Come scrive Zangrandi, esso "presenta un aldilà interiore, psico-patologico e inconscio, attinge dalla cultura e dalla società del suo tempo», aspirando «a una medita- 
zione sugli incubi e sulle tensioni dell'uomo moderno» (2011: 9); la studiosa spiega come i testi fantastici del Novecento, descrivendo una situazione al contempo assurda e possibile, dunque credibile, non puntino tanto a terrorizzare il lettore quanto a lasciarlo attonito e spaesato.

Infine, caratteristica della letteratura fantastica contemporanea, che a mio avviso si rispecchia anche in molta ricerca teatrale, è l'uso di un linguaggio fondato sull'immagine che si avvale della parola per restituire nuove realtà.

Dal momento che l'oggetto che determina il fantastico non può essere rivelato nella sua totalità, è necessario che lo si intraveda appena: occorre perciò ricorrere a giochi linguistici, analogia, comparazioni in modo da renderlo un oggetto esclusivamente verbale. La letteratura fantastica gioca col linguaggio, creando, manipolando e associando immagini, punta sulle sue facoltà creative in quanto solo le parole possono creare una nuova e diversa realtà. Il modo fantastico utilizza le potenzialità del linguaggio per caricare di valori le parole al fine di creare la sua realtà, ricca di contraddizioni e incontri degli opposti. Il gioco verbale ha la funzione di conferire carattere magico al linguaggio ormai abusato della quotidianità: si giustificano così i frequenti ricorsi a climax, iperbole, ossimoro, ellissi, aggettivazione fortemente connotata, sinestesie e metafore che concorrono a incrementare la visibilità del dettato. Il continuo aggrumarsi e sciogliersi nell'avvicendamento degli avvenimenti creano quel fantastico misterioso, strano, intellettuale, allegorico e metaforico che lascia il lettore perplesso e smarrito. Il fantastico investe il testo anche a livello stilistico e la narrazione mostra i limiti del linguaggio che deve dare un nome a una realtà che non è spiegabile. Per dare voce alle cose del mondo prive di voce, la scrittura si distingue per l'intensa oggettivazione e propone le combinazioni più diverse (prenominali, postnominali, a sandwich, in triade, in elenco...). Il fantastico punta sull'iconicità e sulla figuratività sia tramite specifiche scelte linguistiche, sia nella scelta di elementi tematici che coinvolgono la sfera visiva. (Zangrandi, 2011: 12)

Il fantastico resta perciò «legato al concetto di ambiguità» e ancora una volta conferma la sua stretta relazione con l'Unheimlich nel dare voce a ciò che è celato nell'inconscio, sia esso individuale che collettivo, sgretolando ogni certezza e tentando di conciliare sogno e realtà. «Il fantastico moderno fa dell'uomo stesso l'oggetto inquietante, l'uomo, con i suoi sogni, le sue allucinazioni, le sue pulsioni inconsce, le sue incertezze, i suoi smarrimenti; nel fantastico novecentesco l'uomo ordinario diventa essere straordinario e l'eccezione diventa la regola» (Zangrandi, 2011: 45). Esso si rivolge alle aree di frontiera nell'uomo, scavando nella sua vita interiore e facendo emergere nuove sensazioni dietro la spinta di esperienze perturbanti. 


\section{Destrutturazione E SOSPENSIONE}

Mi ricollego alle considerazioni di Zangrandi circa la scrittura fantastica per riflettere su come anche il teatro di ricerca abbia imboccato la stessa strada intrapresa dalla letteratura: se infatti il fantastico sulle scene ottocentesche era prevalentemente di carattere narrativo, oggi non lo è affatto e la ricerca teatrale ricorre invece a un uso sempre più metaforico delle immagini, spesso anche con l'aiuto di un montaggio che segue canoni non più vincolati a una successione cronologica o diacronica. Una prima considerazione va fatta allora sull'uso del linguaggio di un teatro che, come ricordavo nell'introduzione, non è più finalizzato alla sola rappresentazione dell'opera drammaturgica e che si crea piuttosto sulla base di nuovi principi.

[...] la composizione scenica, cioè la drammaturgia o scrittura dello spettacolo, ha cercato di modellarsi su principi e procedimenti nuovi: anti-narrativi, anti-mimetici, anti-illustrativi, che hanno messo in crisi, sia pure in misura diversa a seconda dei casi, capisaldi della rappresentazione teatrale, sia registica che pre-registica: la compiutezza unitarietà diegetica, insomma la fabula, il personaggio, la finzione stessa. (De Marinis, 2010: 15)

Una decostruzione/destrutturazione della forma messa in scena che, come spiega De Marinis (2013) fa parte di un fenomeno (performativizzazione del teatro) sorto negli anni Sessanta e tutt'ora in atto, e che porta il teatro a trasformarsi in una vera e propria arte della visione 8 (Mango, 2010). Un teatro, quello postdrammatico, che chiama lo spettatore a farsi nello stesso tempo interprete attivo nel contribuire alla creazione dello spettacolo con la sua visione soggettiva a darne dunque la sua personale lettura. Ed è proprio su questo piano, a livello formale - richiamando le osservazioni proposte dalla critica letteraria e da Freud - che è forse possibile rintracciare una presenza dell'Unheimlich in chiave fantastica. La pluralità di codici e dispositivi di cui il contemporaneo teatro di ricerca si avvale - insieme a un montaggio che, come ho già ricordato, si rivela anti-narrativo - diventa talvolta fonte di una sensazione di spaesamento nello spettatore. Si torna pertanto a quell'esitazione, a quell'incertezza intellettuale a lungo discussa, di fronte all'impossibilità di definizione e alla difficoltà di riconoscimento della realtà dell'opera inscenata: una delle caratteristiche ricorrenti è infatti quella di non rappresentare

8 «[...] sostenere che il teatro moderno è arte della visione non significa solo che gli elementi scenici hanno un peso e uno spazio diversi, ma che diventano il fondamento attorno a cui ruota la dimensione drammaturgica» (Mango, 2010: 41). 
una ben precisa realtà, quella della scena o del dramma. Potremmo considerare fantastica-perturbante l'esperienza dello spettatore proprio perché gli spettacoli a cui assiste sembrano costruiti su un continuo andirivieni tra realtà parallele, in alcuni momenti più vicine alla nostra, fantastiche ed oniriche in altri. Seguendo tale ragionamento non possiamo tuttavia limitarci a definire fantastico un certo filone del nostro teatro di ricerca ma credo sarebbe invece più corretto ipotizzare la condivisione, da parte del teatro, di alcuni principi compositivi adottati dal fantastico, siano essi consapevolmente o inconsapevolmente impiegati per la scena. Si pone quindi un problema di definizione del fantastico nei confronti di una ricerca teatrale che nella maggior parte dei casi nasce da ben altri presupposti tra i quali, l'unico (per ora) rintracciabile e in comune con la forma del fantastico tardo novecentesco e contemporaneo, è forse il desiderio di colpire il proprio pubblico, suscitare in esso determinate sensazioni-reazioni per mezzo di un sapiente montaggio e della scelta di immagini-archetipo che risveglino la memoria, collettiva o personale. Attraverso gli stessi meccanismi appartenenti all'Unheimlich letterario si configura, come reazione, un perturbamento reale nello spettatore: in un teatro che fantastico non è, sono portati in scena i frutti di ricerche che per inevitabile necessità si rivolgono, in molti casi con un linguaggio metaforico, alla vita a agli incubi che la popolano.

\section{UNA QUESTIONE ANCORA APERTA}

Un secondo ragionamento credo vada rivolto anche alle tematiche sulle quali si articolano le forme del fantastico. Anche in questo caso infatti è possibile tracciare un parallelo tra letteratura e teatro contemporanei, rifrazione entrambi di un malessere individuale e collettivo e specchio della riflessione dell'uomo su se stesso, dove il fantastico si propone come strumento di meditazione sulle sue tensioni e sui suoi incubi. Ma non solo, il fantastico letterario, che diviene esortazione a una considerazione sulla società e sul tempo presente, «è anche il mezzo per irridere certi comportamenti umani e per fare una severa critica alla società usando uno strumento che tenta di conciliare realtà inconciliabili» (Zangrandi, 2011: 48).

Come accade in ambito letterario anche nell'ultimo teatro di ricerca si scorgono elementi ascrivibili al mondo del fantastico, parti di un campionario che vede materializzare sulla scena figure mitologiche, mostri, esseri nati dall'ibridazione tra uomo e animale o tra uomo e macchina. Nell'intreccio delle diverse realtà drammaturgiche che popolano la scena emergono, a in- 
frangerne i presupposti di esistenza, figure perturbanti ricollegabili al più comune immaginario fantastico. Sono immagini, quelle di cui il teatro sembra avvalersi, che risvegliano l'inconscio dello spettatore rimandando agli incubi più comuni e condivisi del nostro tempo: esseri fantastici ed atmosfere oniriche, resi tali grazie ad un sapiente lavoro drammaturgico e di composizione scenica, all'uso di trucco, costumi, luci, suono, apparati scenici e, non da ultimo, al montaggio. A mio avviso tutti questi sono elementi che possono essere associati tanto all'iconografia del fantastico quanto alla necessità di un uso metaforico del linguaggio, scelto per approfondire tematiche che nulla hanno a che vedere con questo modo o genere: c'è da chiarire allora se le immagini che vediamo sulle nostre scene siano ascrivibili o meno alla forma fantastica. Finora si è dato per definito un immaginario fantastico letterario, ma la stessa critica ha più volte evidenziato come le stesse immagini che popolano oggi racconti e romanzi appartenessero già alle culture, mitologie e religioni più antiche. Insomma, anche il fantastico (come pare il teatro) si nutre di archetipi che da tempo fanno parte della nostra cultura e che possiamo dunque riconoscere. Si torna così agli archetipi collettivi junghiani, a una memoria iconografica tramandata culturalmente e spesso affidata all'inconscio: «anche quando volutamente il narratore insiste sul carattere di irrealtà dell'essere fantastico, mantenendolo nello spazio dell'ambiguità e dell'evanescenza, non può fare a meno di ricorrere a forme che si possono pensare, a particolari che in qualche modo ricadono nell'ambito dell'esperienza comune» (Di Natale, 1995). Le immagini dunque «accadono» (Belting, 2009): l'immagine che vediamo non è altro che la risultante di una somma dell'immagine percepita e quella ricordata, di immagini mentali e immagini materiali, ed è proprio grazie a questo principio che determinate scene a cui assistiamo a teatro agiscono sul nostro inconscio provocando un effetto perturbante. L'azione scenica guida l'attenzione dello spettatore verso la costruzione di una determinata immagine: come sottolinea Belting, «nessuna immagine visibile giunge a noi senza una mediazione. La loro possibilità di essere viste dipende dalla specificità del medium in cui si trovano, che ne regola la percezione e determina l'attenzione dello spettatore» (2009: 77). Seguendo un approccio di tipo antropologico, lo studioso spiega in questo modo come figurazioni mentali e materiali possano essere considerate le due facce della stessa medaglia e come pertanto sogni e icone siano interdipendenti.

L'iconografia fantastica si avvale dunque di tutta una serie di rappresentazioni proprio perché esse hanno il potere di agire sull'inconscio (nel quale le categorie logiche tradizionali sono abolite) e sull'emotività del lettore; 
operazione del resto attuata anche dal teatro di ricerca, il quale spesso si serve di immagini molto forti, che talvolta leggiamo come perturbanti, e che mirano a stimolare una reazione nello spettatore e a creare una effettiva esperienza di disorientamento. In teatro questo elemento straniante e fantastico, se inserito nella giusta cornice, diviene uno strumento di cui si servono dramaturg e regista per raggiungere il pubblico e colpirlo.

Attraverso alcune mie osservazioni (e la personale esperienza di spettatore), ho individuato il delinearsi di due dialettiche che si manifestano ricorrenti in spettacoli o scene che a mio avviso suscitano un'esperienza perturbante nella sua connotazione di fantastico, umano - disumano e assenza - presenza, dalle quali vorrei partire per una lettura delle immagini che sono qui a proporre.

1) umano - disumano

In tal modo, gli esseri viventi acquistano progressivamente attraverso variazioni successive tutti i caratteri che di essi ci sono noti, e la superficie coerente e solida da essi formata non è, qualora vengano considerati nella dimensione del tempo, che il risultato frammentario d'un continuo assai più serrato, assai più fine: un continuo che è stato tessuto con un numero incalcolabile di piccole differenze dimenticate o abortite. Le specie visibili che si offrono alla nostra analisi sono state ritagliate sullo sfondo incessante di mostruosità che compaiono, scintillano, si inabissano, e talora si conservano. Questo è il punto fondamentale: la natura ha una storia solo nella misura in cui può contenere il continuo. Proprio perché assume di volta in volta tutti i caratteri possibili (ogni valore di tutte le variabili) essa si presenta nella forma della successione. (Foucault, 1970: 172)

Elementi ricorrenti nella letteratura fantastica sono il mutante, l'ibrido (tra uomo e animale o tra uomo e macchina), il mostro: figure che in diverse varianti riscopriamo anche nel teatro contemporaneo, ${ }^{9}$ dove corpo e corporeità diventano in maniera sempre più preponderante elementi centrali della relazione attore-spettatore.

Nel suo saggio dedicato alla letteratura, Todorov (2007 [1970]) introduce quello che definisce il tema dell'io, legato alla metamorfosi e all'esistenza di esseri soprannaturali e al loro potere sugli uomini; concetto per il quale egli

9 Metamorfosi inscenate con il sostegno di apparati tecnologici; automi ed esseri meccanici; ibridazioni tra uomo e animale, ottenute con stratagemmi ostentati o celati; attori che si avvalgono dell'ausilio di protesi meccaniche, o che presentano la loro naturale anormalità portando in scena i loro corpi diversi. 
teorizza che la rottura del limite tra materia e spirito sia pretesto per incessanti trasgressioni, le cui conseguenze possono essere la moltiplicazione della personalità e l'annullamento del confine tra soggetto e oggetto. Una delle provocazioni più ricorrenti nella letteratura fantastica, e fonte di una sensazione perturbante, sembra essere dunque quella di una minaccia al sé, un attacco che «lo rende parte dell'altro», con una successione di fasi che vanno dall'invasione, alla metamorfosi, alla fusione (Jackson, 1986).

Nell'arte moderna e contemporanea l'essere anormale, il mutante, è quindi talvolta metafora di una scissione interiore che può essere controllata grazie alla sua proiezione esterna (Di Natale, 1995). La metamorfosi, in particolare, sovverte il principio base di identità ed esprime la paura primaria di dissoluzione e non esistenza (Fusillo, 2004-2007). Braidotti, riferendosi all'origine del mostro e ripercorrendo la storia della teratologia, spiega come vi siano differenze d'ordine morfologico che vengono definite come mostruosità.

I corpi «deformi» sono infatti una costante non solo del discorso scientifico, specialmente quello biologico, psicologico e medico, ma anche di molte discipline sociali che puntano sulla «normalità» come modello di base. L'anomalo, o l'anormale, viene dunque costituito come figura abietta e tuttavia ubiqua: è l'altro che si deve in ogni modo evitare, quello a cui è proibito assomigliare. Tutto si gioca appunto sulla somiglianza e la differenza, dove i segni distintivi e quindi i criteri di differenziazione non sono gli attributi sessuali, ma organi, morfologie e fisiognomie specifiche. La normalità definita come «grado zero della mostruosità» riassume in sé una serie di aspettative e di norme socio-simboliche che fanno di un certo tipo di corporeità il modello di base. (Braidotti, 2005: 10)

La studiosa spiega come oggi la questione delle mutazioni genetiche sia stata trasferita dai laboratori scientifici alla cultura popolare, innalzando la metamorfosi ad icona, sottolineando come il mostro incarni anche l'intermedio, l'ibrido, l'ambivalente, la deviazione e l'anormalità (2005: 29). Braidotti ne elenca alcune categorie: differenza sessuale (ermafroditismo); razza ed etnia; il non-umano, sia nella veste di sacro e divino che nella variante dell'animale, del degenerato e del mutante; l'altro inorganico, ovvero il replicante tecnologico, la macchina. La figura del mostro, essendo metamorfosi dell'uomo normale, si colloca allora nella zona intermedia tra ciò che è estraneo e ciò che è familiare, presupposto per cui l'immagine della mostruosità per-turba l'osservatore. Insomma, nell'ibrido convivono gli estremi opposti: umano ed animale, infimo e superiore, animalità e divinità (Vergine e Verzotti, 2004). 
In una cultura come la nostra, che identifica il reale con il visibile, l'irreale è associato all'invisibile: come spiega Jackson (1986), la vacuità dei mondi del fantastico altera un mondo percepibile insistendo sull'assenza. Si presentano allora universi vuoti, buchi nella realtà: tempo e spazio sembrano spesso sospesi o prolungati oltre il possibile; si verificano inversioni dei domini di sogno e realtà (Caillois, 1984 [1965]); si tende verso la sospensione del tempo, non più cronologico, in una dimensione di eterno presente.

Anche il tema dell'occhio e delle forme del vedere è soggetto ricorrente nel fantastico, quello della visione e della visibilità è argomento affrontato in letteratura con il ricorrere a immagini spettrali, specchi, vetri, occhi, lenti, riflessi: il perturbante è feticistico, è il vedere cosa non può essere visto (Sánchez-Biosca, 1999). Sono allora percepite come perturbanti tutte quelle situazioni in cui lo spettatore ha la visuale impedita da qualcosa (oggetti, apparati scenici, oscurità); gli elementi che sono destinati a offrire rappresentazioni, ma che le rifiutano, le nascondono, le evitano grazie alla loro posizione o alla loro distanza; un apparato che mostra ciò che non è visibile allo spettatore, come una proiezione su uno schermo o uno specchio: «anziché indugiare presso gli oggetti visibili lo specchio attraversa l'intero campo della rappresentazione trascurando ciò che potrebbe captarne, e restituisce la visibilità a ciò che si manifesta fuori da ogni sguardo» (Foucault, 1970: 22).

La delineazione di una cornice, visibile o invisibile, diventa infine sia espediente per focalizzare l'attenzione su una determinata immagine o scena che mezzo di ostensione e voyeurismo: «windows are at the same time textual devices aimed at producing suspense and mystery, or at framing extraordinary events; and thematic elements, linked to nostalgia of the elsewhere, to oneiric contemplation» (Fusillo, 2004-2007: 1).

\section{LA PAROLA ALLE IMMAGINI}

Non potendo in questa sede affrontare una completa catalogazione e un'esaustiva analisi di tutti gli elementi discussi, vorrei proporre alcune immagini che appartengono alla più recente produzione teatrale italiana che a mio avviso riflettono in un estetismo perturbante le due dialettiche suggerite. Anche se purtroppo poche fotografie non possono essere efficaci rappresentanti di una messa in scena, ne propongo di seguito alcune insieme a dei materiali riferiti a due progetti e due spettacoli portati sulle scene da alcune delle 
più importanti compagnie italiane: il ciclo della Tragedia Endogonidia (20022004) e Giulio Cesare (1997), della Sociètas Raffaello Sanzio; il progetto O - Z (2007-2010), di Fanny \& Alexander; Paesaggio con fratello rotto (2004), del Teatro Valdoca.

In conclusione, la questione se esista o meno un teatro fantastico del nuovo millennio rimane, per il momento, ancora del tutto aperta.

Paesaggio con fratello rotto

Trilogia: Fango che diventa luce, Canto di ferro, A chi esita ${ }^{10}$

La trilogia porta in scena una meditazione sul rapporto dell'essere umano con il mondo, la denuncia verso l'indifferenza dell'uomo per l'ambiente a cui appartiene e riflette sulla nostra «umanità contraddittoria che è ad un tempo vittima e carnefice». Al confine tra narrazione e anti-narrazione, la scena dà vita a situazioni e figure indiscutibilmente fantastiche, innescate dai mutamenti del non-luogo drammaturgico con apparati scenici che cambiano continuamente la loro significazione (il tavolo d'acciaio e vetro sul fondo della scena del primo «paesaggio» è sia bancone da macellaio che altare sacrificale), ma date anche dalla natura dei personaggi, esseri antropomorfi o deformi: la Ragazza uccello, la Ricamatrice, il Ragazzo cane, la Geisha di Canto di ferro sono "figure eccessive, rubate a un dionisiaco bestiario e ricondotte a una mitologia personale» (Ponte di Pino, 2006). Infine, giocano la loro azione perturbante alcuni contrappunti generati da voce e gestualità, dall'opposizione tra la voce affettata dell'Oracolo e la sua potente presenza scenica come dal silenzio degli «animali» unito alle loro movenze sincopate e spezzate.

In quest'opera [...] c'è un dolore che sembra riguardare soprattutto l'occidente: la spaccatura micidiale fra noi e l'anima del mondo, quell'energia intuita e sempre tradita, che ci tiene vivi.

Questa «anima del mondo», taciuta con superiorità dalla scienza, rimpicciolita a corpuscolo con macchie dalla religione, resa ridicola dalla razionalità, resa retorica e melensa dalla lingua corrente, questo pezzo di brace cosmica che brucia nella terra e in ognuno di noi, questo è ciò che viene fotografato in quest'opera.

[...]

10 Regia e luci Cesare Ronconi, parole Mariangela Gualtieri. Sul sito ufficiale della compagnia sono pubblicati i materiali del progetto: http://www.teatrovaldoca.org. Le fotografie, di Rolando Paolo Guerzoni, qui pubblicate sono state gentilmente concesse dalla compagnia. 
Un'onda acustica strappa lo spettatore dalla terra ferma e lo trascina dentro un paesaggio umano, dentro un fratello rotto nella sua polarità che pare inconciliabile, fra la forza spirituale e la forza bruta. Dai corpi arriva tutto l'ibrido che la regia ha evocato e composto, tenuto in equilibrio sottile: vigore e spegnimento, fecondità e arsura, infanzia e crudeltà, candore e seduzione, bellezza e dolore, sterilità e dono di sé. Guardo le sette figure in scena ${ }^{11} \mathrm{e} \mathrm{mi} \mathrm{ap-}$ pare la campionatura di un'umanità a venire, così imperfetta, così amabile $\mathrm{e}$ misteriosa. Sono estremizzate nel loro essere tipi iconografici, reali e fantasmatici, mossi da energie tremendamente antiche.

[...] Le parole sono lontane dall'atlante che immagini compongono, lontane eppure appropriate, come echi interiori di un io profondamente senziente e sapiente in un corpo che simula tutt'altro. Non più le voci adulte della prima parte: da queste gole esce un dire piccolo e incerto, eppure savio, profondo, accorato. Così la geisha orgiastica e sterile, che da cortigiana si fa neonata, porta la propria voce riflessiva, sperduta e dolce, come un fagotto antico.

O la ragazza uccello, nel suo slancio al volo, fatto di nevrosi e tenerezza, che lancia la sua lettera d'amore con disarmante dolcezza. O il ragazzo cane, Cerebro furioso e poi mite, che biascica contro la «solfa dei dolori umani». (Gualtieri, 2004)

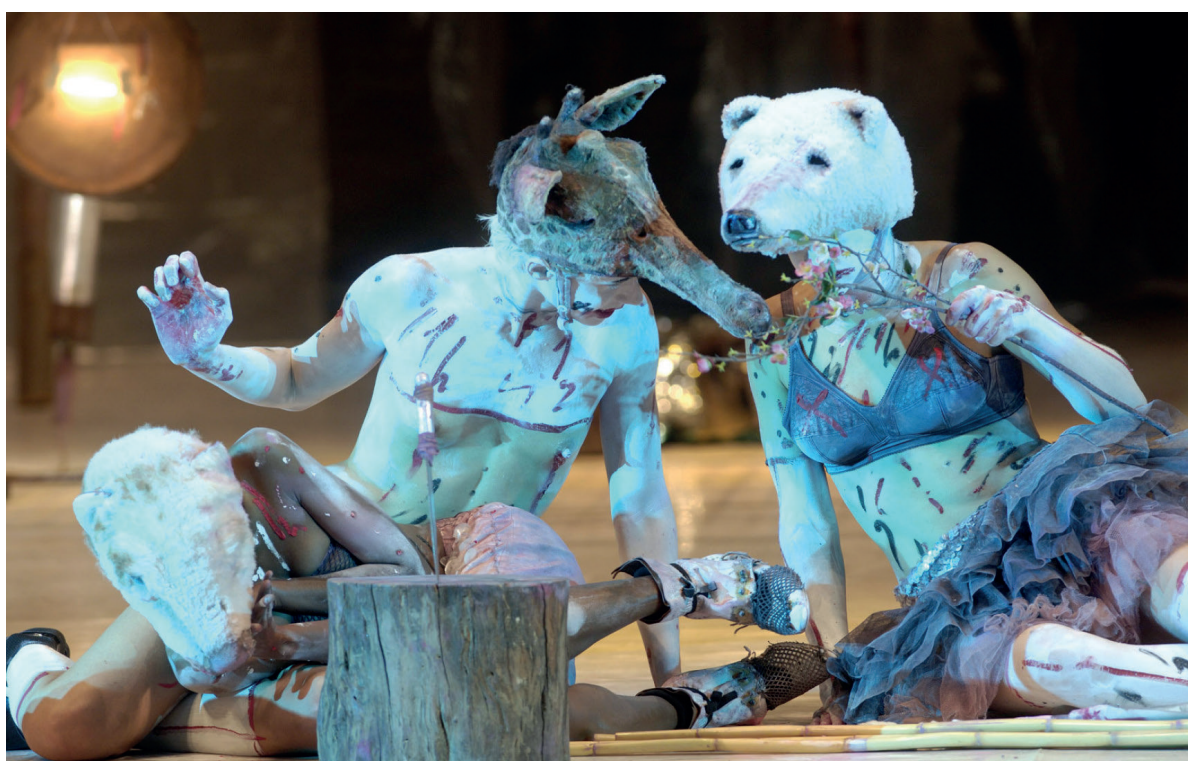

1. Fango che diventa luce

11 Questa parte del testo è una nota della seconda tappa dello spettacolo, Canto di ferro. 


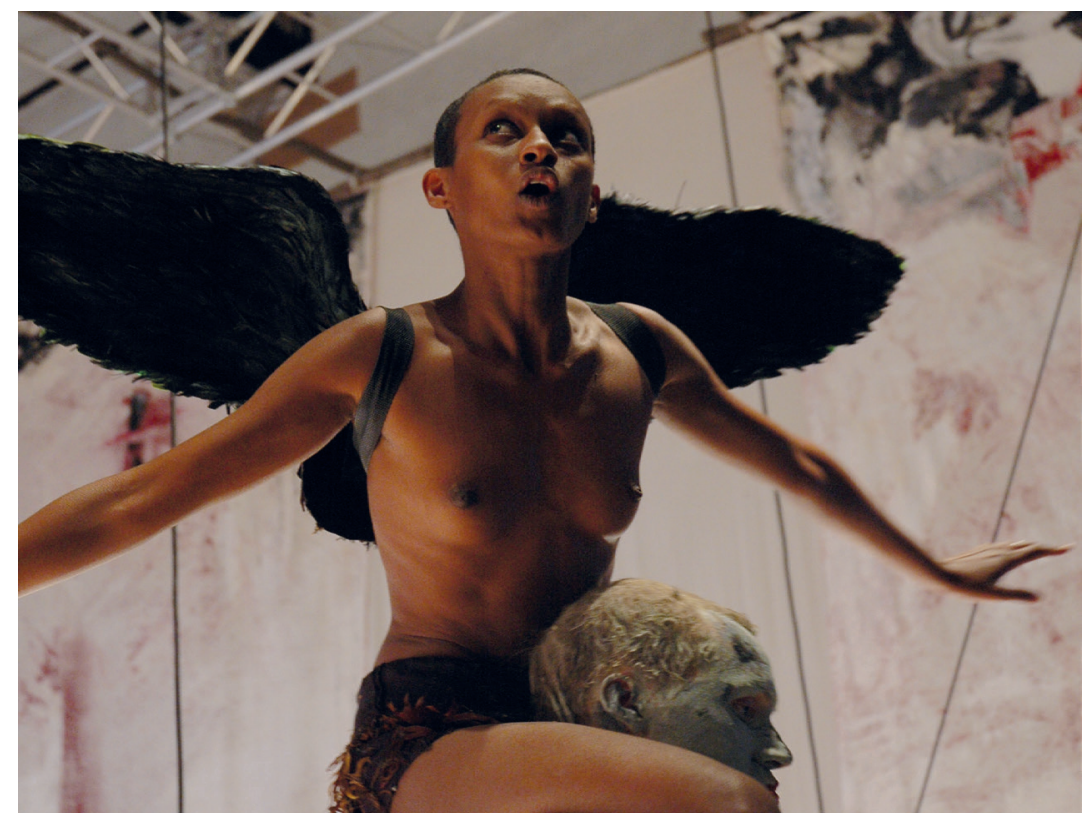

2. Canto di ferro

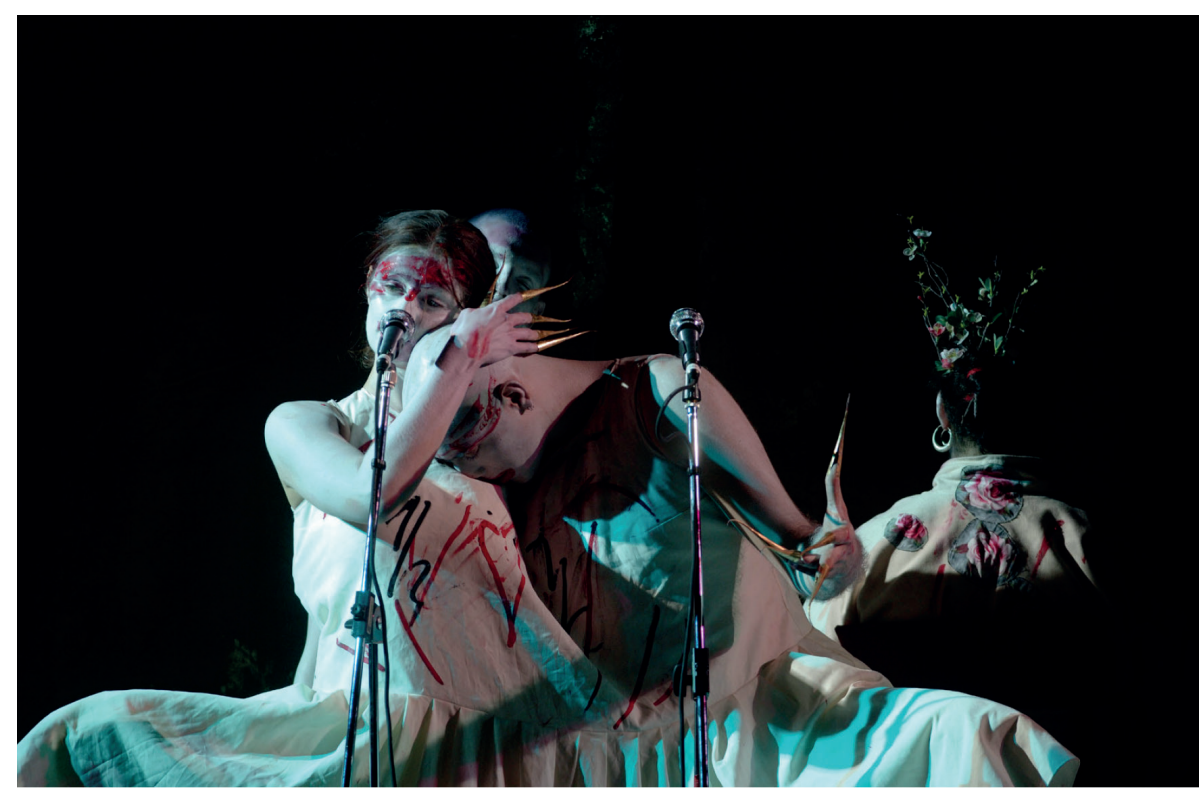

3. A chi esita 
$O-Z^{12}$

Il progetto è metafora di un viaggio in cui lo spettatore è protagonista di un'avventura che lo conduce sui passi di Dorothy lungo le tappe del suo cammino verso la città di Smeraldo, in un cammino immaginario che va dal North degli incubi della mente al South degli smarrimenti dell'anima. Costruito a partire dal romanzo di L. Frank Baum Il meraviglioso mago di Oz (The Wonderful Wizard of Oz, 1900), esso è un viaggio che, guidato da artisti diversi e passando da Kansas a Emerald City e ritorno, via est, sud, nord, ovest nelle sue tappe si dispiega in un labirinto di significati, immagini e allusioni riassunti in alcuni temi-archetipo come coraggio, attesa, scelta.

Dorothy parla su un palcoscenico nudo, in una scenografia di luci e suoni, dietro una lente deformante che altera il suo aspetto e la trasforma di volta in volta nella bambina con le scarpette rosse e nel suo cupo e ambiguo interlocutore Oz. Sono gli apparati scenotecnici qui a dare vita a figure perturbanti: ora è la lente-frame che ostenta l'aspetto mostruoso del mago, ora la luce diffusa dietro la quale si staglia l'ombra di uno spaventapasseri spettrale che si muove in lontananza, avvolto da una coinvolgente atmosfera sonoro-luminosa.

Trattasi di uno spettacolo di teatro musicale o scène lirique dove, a partire dalla favola di L.F. Baum, si ripercorrono le avventure della leggendaria Dorothy nel regno dell'inautentico, del meraviglioso, del fantastico mondo di Oz. Oggi il disastro è sotto gli occhi di tutti. Il ciclone, l'uragano è in agguato, basta accendere la tv: e non è solo metereologico... Ma possono la musica e il teatro, oggi, procurarci un altro, più intimo e segreto ciclone, capace di trasportarci in una dimensione come quella del meraviglioso mondo di Oz? O farci porre domande rispetto al mondo con la stessa innocenza di una Dorothy? Un universo parallelo così caleidoscopico e iridato, utopico, dove le risposte ai quesiti del mondo non sono in bianco e nero, ma variegate e fluide, ambivalenti e incongrue e la lingua che si utilizza non è mai univoca, non può che essere evocato dal potere sinestetico della musica e del teatro. Ma è possibile ricostruire il fantastico di fronte alle pieghe rovinose del nostro tempo?

$[\ldots]$

12 Ideazione Luigi de Angelis e Chiara Lagani, regia, scene, luci Luigi de Angelis, drammaturgia Chiara Lagani, testi Chiara Lagani, Fiorenza Menni e Francesca Mazza. Sul sito della compagnia sono disponibili i materiali degli spettacoli: http://www.fannyalexander.org. Le immagini qui riprodotte, di Enrico Fedrigoli, sono state gentilmente concesse dalla compagnia. 
«South» sprofonderà Dorothy, e con lei lo spettatore, in una cupa notte, un buio percussivo e vorticoso, da cui nascerà una nuova lingua, quella ritmica, del battito, la lingua lunare animalesca e notturna della Strega di quel vento, una creatura nata forse da quel mondo sonoro in cui Dorothy si è immersa e trasfigurata.

[...]

La protagonista, Dorothy, un avatar dello spettatore, è catapultata dal suo ciclone a Sud e poi a Nord dell'universo fantastico attraverso cui il suo viaggio-avventura si svolge.

Entrambi gli spettacoli [North, South] prendono mossa dalla famosa domanda del Mago: «Sono Oz il grande e terribile e tu chi sei?» (Fanny \& Alexander 2014)

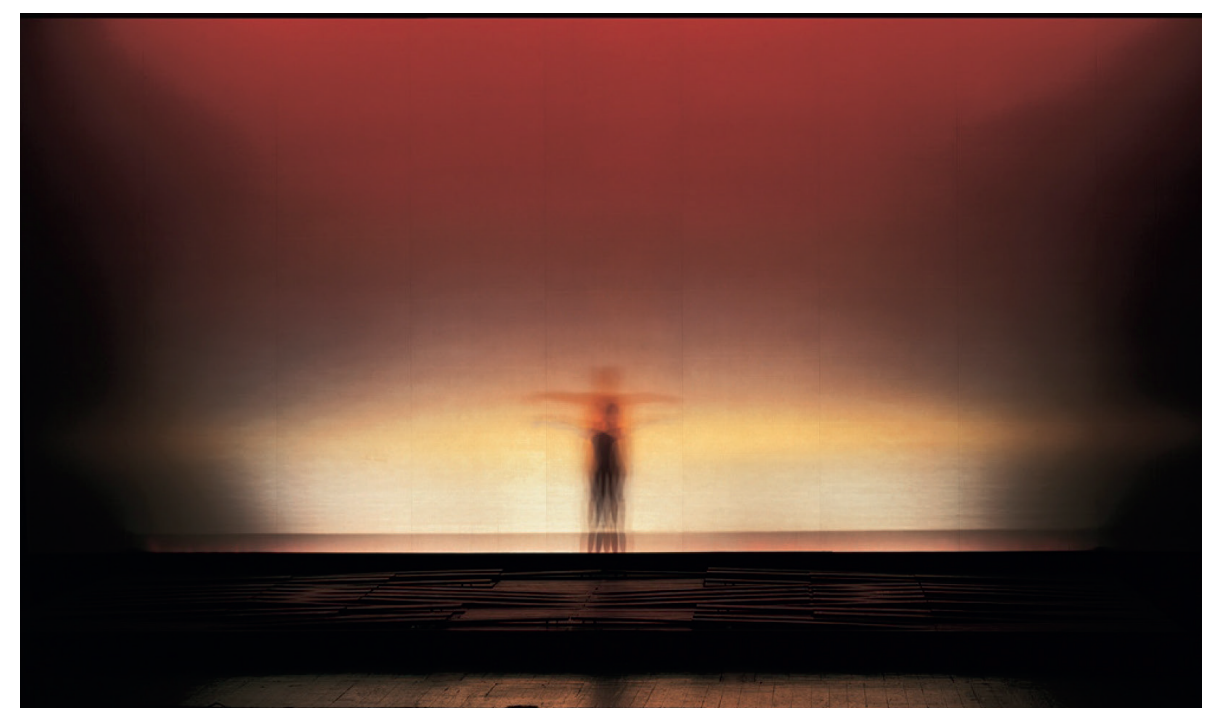

4. North. Incontro con lo spaventapasseri 


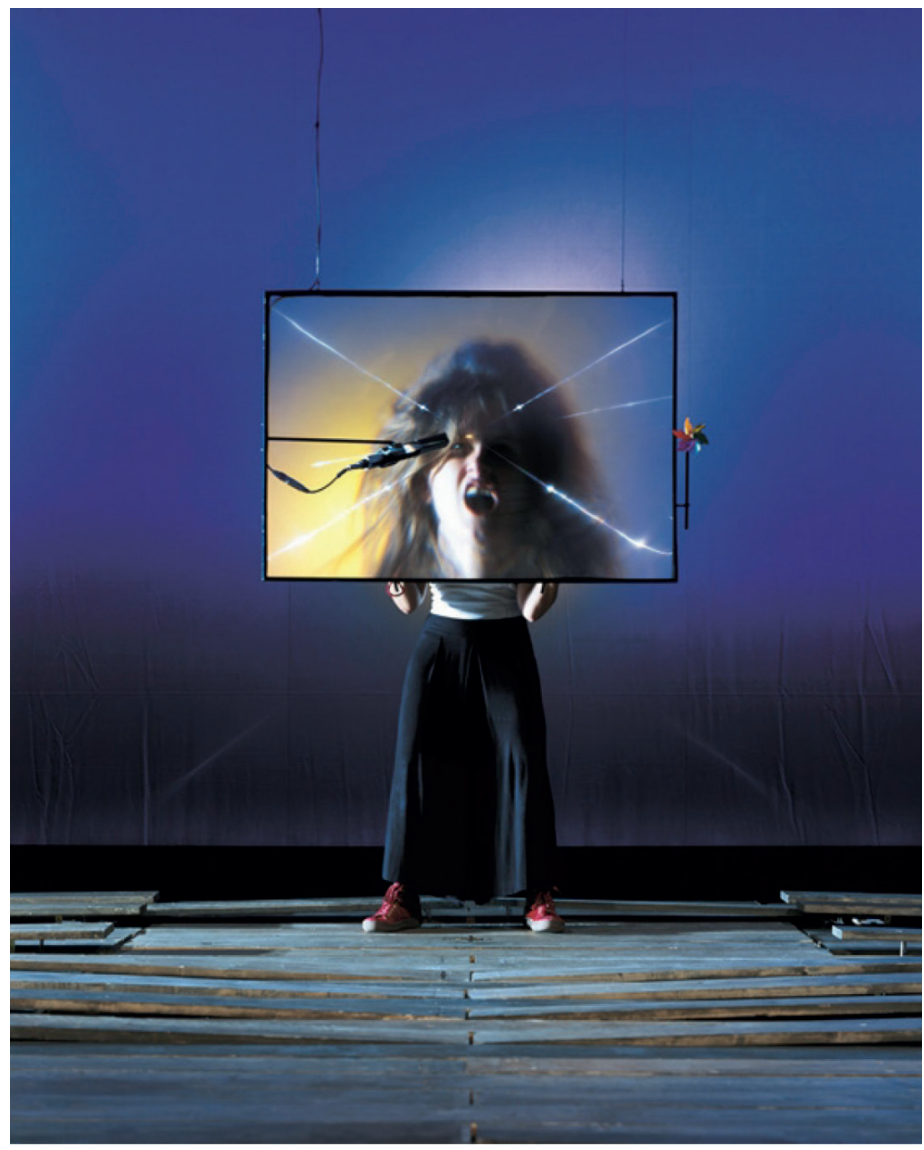

5. North

\section{Giulio Cesare ${ }^{13}$}

Lo spettacolo, che nasce da una rivisitazione della tragedia di Shakespeare, vede come protagonisti dei non-attori, persone che portano sulla scena la loro particolare fisicità: Cesare è un vecchio, Cicerone un obeso incappucciato, Antonio un laringectomizzato, Bruto e Cassio due ragazze anoressiche. Obesi, freaks, corpi brutalizzati o mutanti in una costante metamorfosi tra uomo e animale: sono gli archetipi ricorrenti nel percorso della

13 Regia e suono Romeo Castellucci. Sul sito della compagnia, http://www.raffaellosanzio.org, è presente la scheda tecnica dell'ultima produzione di Romeo Castellucci, Giulio Cesare. Pezzi staccati, debuttato nel Marzo 2014 a Bologna. Le immagini, gentilmente concesse dalla compagnia, sono riferite invece allo spettacolo del 1997: PhotoCredits: SRS. 
Societas Raffaello Sanzio alla ricerca di un personalissimo teatro della crudeltà in cui il segno teatrale si riappropria della sua potenza destabilizzante in un tragitto verso il mito, il pre-umano e l'animale. Dai primi spettacoli (Amleto. La veemente esteriorità della morte di un mollusco, 1992; Giulio Cesare, 1997), la scena si anima d'immagini che tendono a disorientare lo spettatore portando all'estremo la dis-umanizzazione o l'iper-umanizzazione del corpo. L'apparizione di mostri-mito richiamano immagini archetipiche alla coscienza dello spettatore, costringendolo a farsi testimone del divenire e attore allo stesso tempo.

Bruto e Cassio nel secondo atto sono corpi femminili e anoressici. Bruto e Cassio in Shakespeare sono presentati come due generali sui campi di battaglia, e per me i corpi anoressici sono corpi militareschi, armati, che conducono una battaglia feroce nei confronti di se stessi e, tra l'altro, Bruto e Cassio sono due generali sconfitti e i corpi anoressici sono corpi sconfitti, e come tali corpi perfetti. Così come, a mio avviso, sono perfetti i 180 chili di Cicerone e la vecchiaia decrepita di Giulio Cesare o Antonio, che è la voce retorica, che vince e che nello spettacolo esce da una ferita (l'attore laringectomizzato). (Castellucci, in Alfano Miglietti, 1998)

All'inizio della seconda parte la scena appare devastata, mentre un maglio ancora picchia nella sua opera di distruzione. Adesso ci si trova per davvero dentro un teatro. (C'era un teatro anche a Hiroshima...) Le poltroncine sono sventrate. Il sipario strappato. Intorno solo rovine. Sul fondo, appena percettibile nella penombra, si muove una figura piccola e scura. Se ne sente soprattutto il rantolo che rompe il silenzio della sala, mentre avanza con un paio di archeologici sci. Si ferma. Si mette in ascolto dei rumori che giungono attraverso le vibrazioni del suolo. Poi il rumore irrompe forte e con la musica entra dal tondo un'altra figura sottile, appesa a un guantone da boxe, ancor più magra della prima e quasi insopportabile alla vista. Bruto e Cassio hanno preso il corpo fragile di due giovani anoressiche, Elena Bagaloni e Cristina Bertini, che inscenano la fase terminale di un'amicizia. La cerimonia di un duplice addio alla vita, inframmezzato dal grido di rivolta dell'attrice contro il pubblico, maledetti è tutta colpa vostra. (Manzella, 2014) 


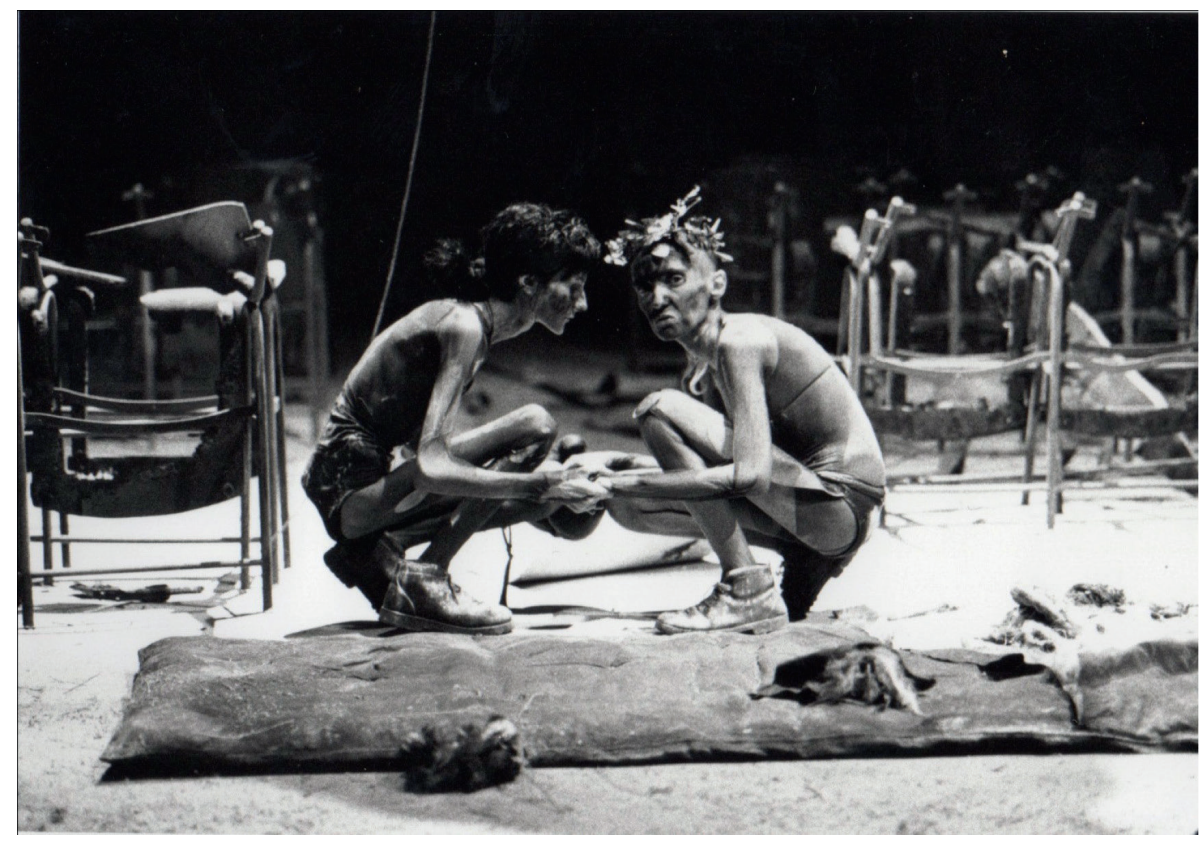

5. Bruto e Cassio

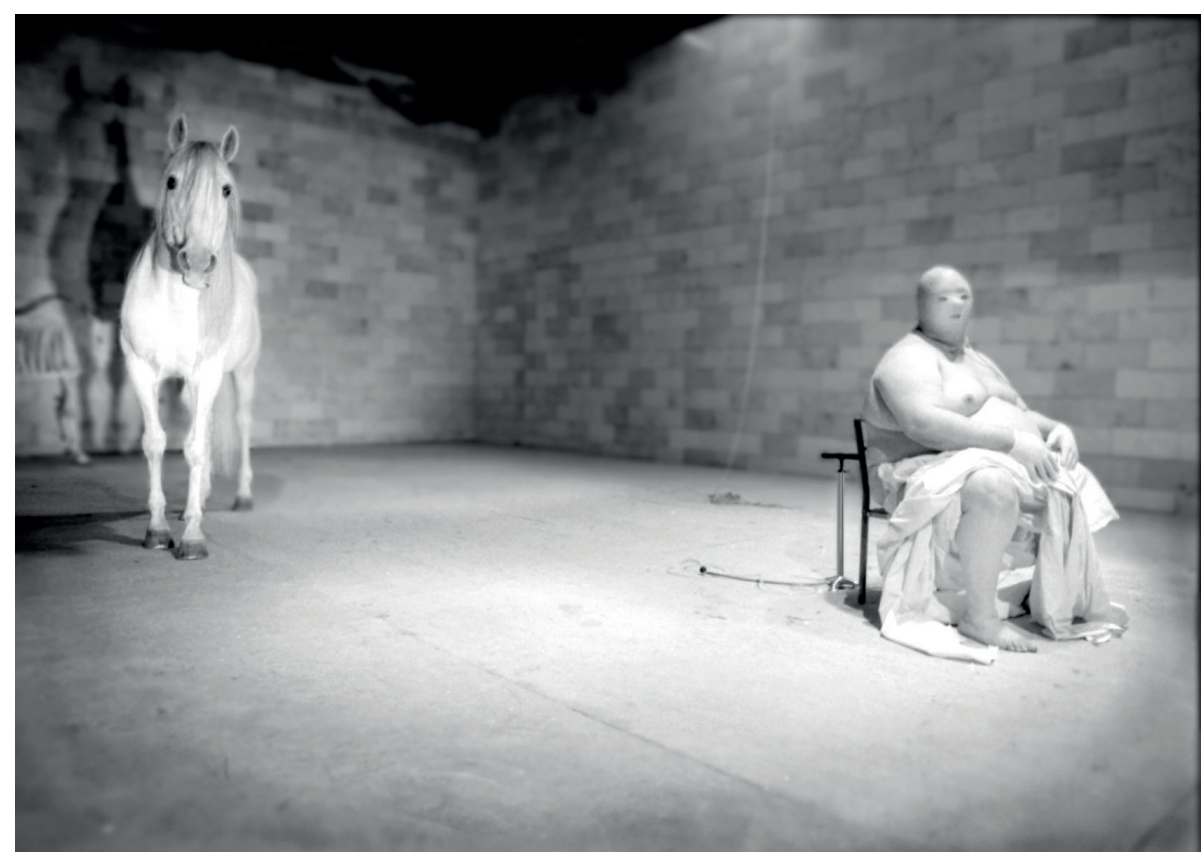

7. Cicerone 


\section{Tragedia Endogonidia ${ }^{14}$}

Il ciclo, della durata di tre anni, ha visto la produzione di undici spettacoli in dieci città europee come parti di un unico corpo organico drammaturgico in continua trasformazione, «un cammino che sospinge verso il mito e ancora di più il rito. Verso il pre-umano e l'animale [...]. E sospinge verso il corpo, il dato elementare della fisicità» (Ponte Di Pino, 2003). Fortemente caratterizzata da un estetismo perturbante, l'intera opera della Raffaello Sanzio rivela un'iconografia che ci immerge in una dimensione onirica, costituita di segni di non facile interpretazione, che ci obbliga a fare i conti con la nostra condizione umana e storica invitandoci ad una riflessione sul mistero dell'uomo nel mondo. Le opere sono popolate di corpi mutanti, incubi visionari e immagini evocate che definiscono una realtà in cui il mostro non è altro che la rivelazione di un'identità nascosta che si moltiplica e trasforma all'infinito.

Il ciclo di lavoro prevede una prima base a Cesena, con l'autogenerazione di una catena di immagini (tragedia endo-gonidia) da cui si distacca una serie di spore che saranno raccolte in altre «basi».

Lo scopo è quello della perpetuazione dell'immagine.

A loro volta le spore daranno vita a unità tragiche singolari.

[...]

Il processo non sarà quello dell'accumulazione, bensì quello della trasformazione vivente. (Castellucci, 2007: 6)

La tragedia nasce esattamente quando l'esperienza del mondo si frantuma, da un livello di contemplazione a un problema di conoscenza.. In una parola nasce in coincidenza della nascita della visione del cittadino ateniese. Visione che non capisce e non accetta più la violenza del rito cruento. Per questo motivo, e da questo momento in poi, la tragedia diventa il laboratorio e l'ambito esclusivo di tutte le violenze. L'alienazione, in un rovescio totale dei valori, diventa in questo ambito la più pura delle possibilità offerte al cittadino-spettatore. Stare in un altro posto. Pensare da un'altra posizione. Spostare lo sguardo all'esterno. La macchina-tragedia sostituisce e sospende ogni altro ordine. L'eroe è il padrone assoluto del proprio corpo solo un momento prima dello schianto. Si libera e esplode nell'attimo finale, nell'attimo in cui è davvero fuori da ogni salvezza e redenzione. Il compito della tragedia del futuro è forse quello di non avere più alcuna idea di corpo suffragata dall'esperienza del sensibile. (Castellucci, 2007: 17)

14 Regia, scene, luci e costumi Romeo Castellucci, regia, partitura vocale, sonora, drammatica Chiara Guidi, traiettorie e scritture Claudia Castellucci. La pubblicazione delle immagini è stata gentilmente concessa dall'autore, Luca Del Pia. 
[...] Le creazioni della Socìetas sono strutturalmente apocalittiche, cioè costruite sull'associazione di apparizioni bestiali e mitico-teofaniche, sulla meraviglia fitta di implicazioni mentali ottenuta con macchine e congegni pertinenti all'officina dell'illusione teatrale. [...] I progetti della Socìetas rovesciano lo scrigno dei miti e delle tragedie, i loci della coscienza condivisa, compilano e sfogliano il bestiario delle storie universali, adottano corpi alterati e minacciati: anoressiche, malati terminali, laringectomizzati, attori addestrati alla confidenza con gli animali, Cani, capre, scimmie. (Guarino, 2001)

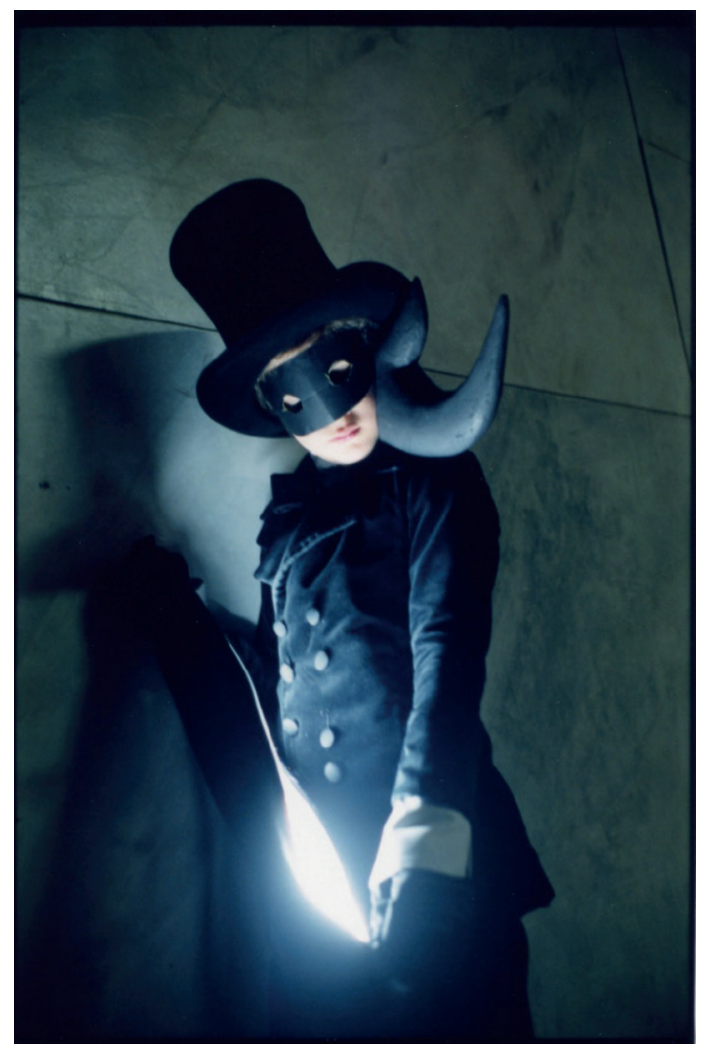

8. BR.\#04 Bruxelles 


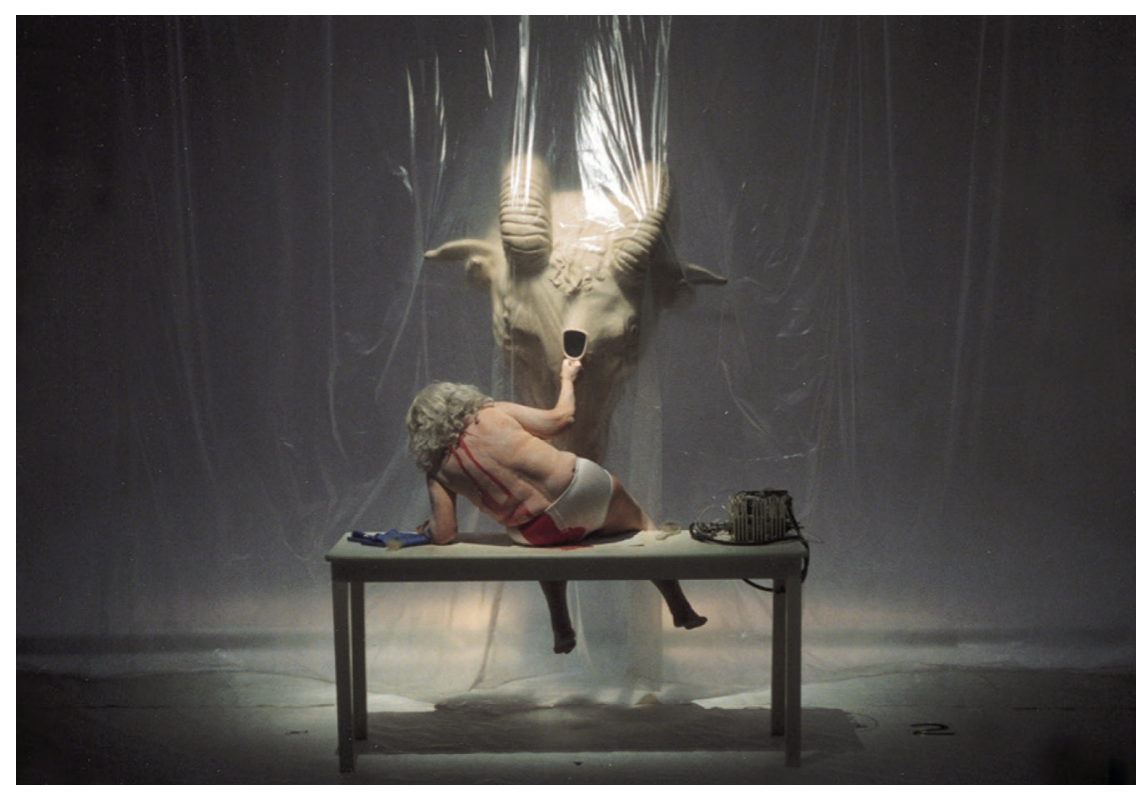

9. BN.\#05 Bergen

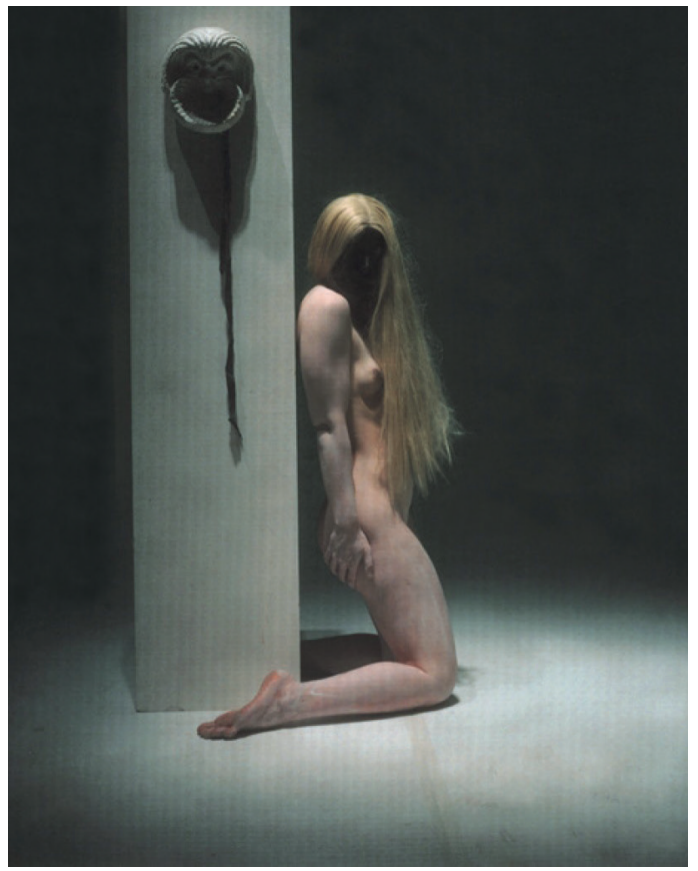

10. L.\#09 London 


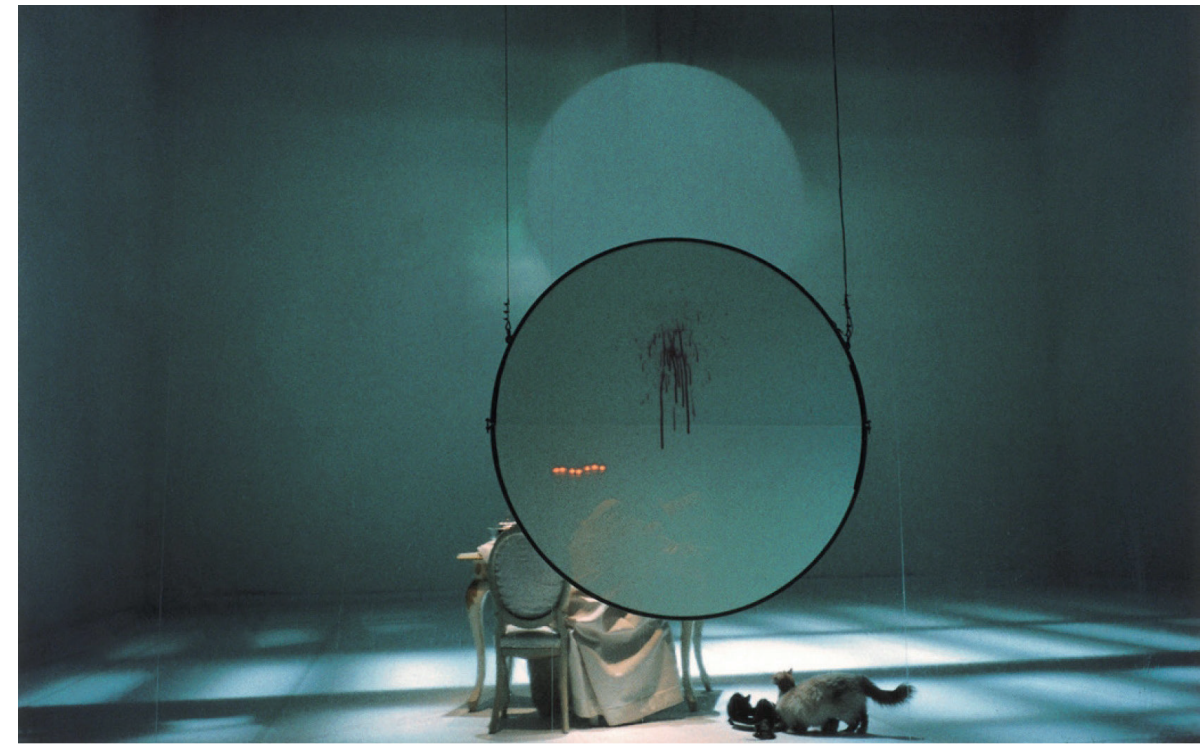

11. L.\#09 London

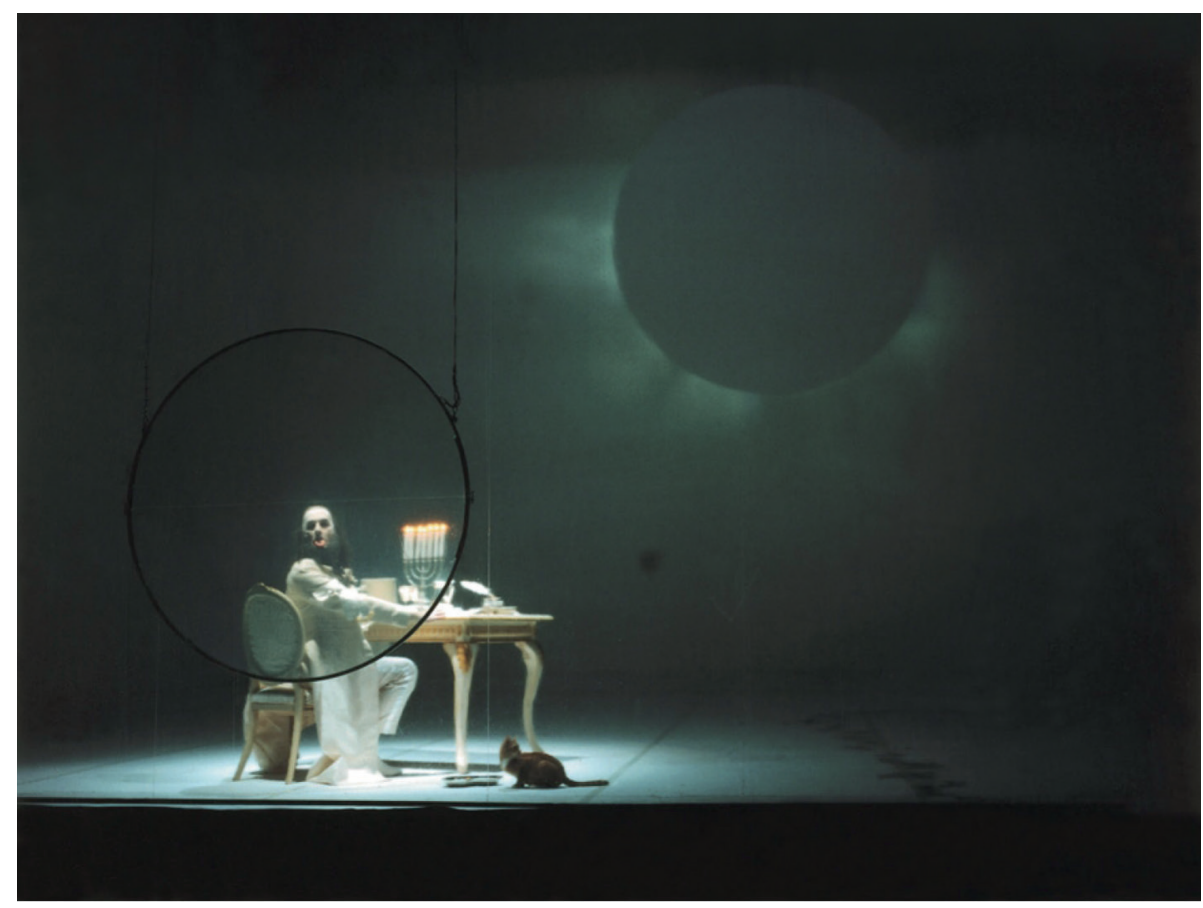

12. L.\#09 London 


\section{BIBLIOGRAFIA}

Alfano Miglietti, Francesca (1998): «Socìetas Raffaello Sanzio - Conversazione con Claudia e Romeo Castellucci», in Virus (1994-1998), n. 14, disponibile in <http:// www.undo.net/it/magazines/933692074> [29/6/2014].

De Angelis, Luigi e Lagani, Chiara (2010): Fanny \& Alexander, O/Z. Atlante di un viaggio teatrale / Atlas of a theatre journey, Ubulibri, Milano.

Antonelli, Emanuele (2010): «Considerazioni mimetiche su Il perturbante (Das Unheimliche)», Enthymema, vol. II, pp. 154-170.

BAL, Mieke (2009): «Leggere l'arte?», in A. Pinotti, A. Somaini (ed.), Teorie dell'immagine. Il dibattito contemporaneo, Raffaello Cortina Editore, Milano, pp. 209-240.

Belting, Hans (2009): «Immagine, medium, corpo. Un nuovo approccio all'iconologia», in A. Pinotti, A. Somaini (ed.), Teorie dell'immagine. Il dibattito contemporaneo, Raffaello Cortina, Milano, pp. 73-98.

Berto, Graziella (1999): Freud, Heidegger. Lo spaesamento, Bompiani, Milano.

BongIorno, Giorgia (2009): «Una luce «senza ristoro d'ombra». La poesia di Mariangela Gualtieri», Italies, n. 13, pp. 445-466, disponibile in <www.italies.revues. org/2734> [29/06/2014]. $<$ http://dx.doi.org/10.4000/italies.2734 >

BraidotTi, Rosi (2005): Madri, mostri e macchine, Manifesto libri, Roma.

BresnicK, Adam (1996): «Prosopoetic Compulsion: Reading Uncanny in Freud and Hoffmann», The Germanic Review: Literature, Culture, Theory, vol. 71, n. 2, pp. 114-132. <http://dx.doi.org/10.1080/00168890.1996.9936560 >

Caillois, R. (1984 [1965]): Nel cuore del fantastico, Feltrinelli, Milano.

Castellucci, Romeo (2007): «La Tragedia Endogonidia», in Socìetas Raffaello Sanzio (ed.), Tragedia Endogonidia, Raro Video, Eccentriche Visioni, pp. 16-20.

Ceserani, Remo (1996): Il fantastico, il Mulino, Bologna.

De Falco, Amelia (2010): Uncanny Subjects. Aging in Contemporary Narrative, The Ohio State University Press, Columbus.

De Marinis, Marco (2013): Il teatro dopo l'età d'oro. Novecento e oltre, Bulzoni, Roma.

(2010): «La prospettiva postdrammatica: Novecento e oltre», Prove di drammaturgia, vol. XVI, n. 1, pp. 12-16.

(ed.) (2010): Rappresentazione - Theatrum Philosophicum, Culture Teatrali, n. 18.

Di Natale, Paola (1995): «La valenza conoscitiva del fantastico», Quaderni del Dipartimento, vol. V, n. 2, pp. 107-139, disponibile in <www.disced.unisa.it/Pubblicazioni/Quaderni/Qua_Vol.05-1995/Q_V05b_107139.htm> [21/06/2014].

FANNY \& AleXANDER (2014): «O - Z», disponibile in <http://www.fannyalexander. org/archivio/archivio.it> [29/06/2014].

FARNeTti, Monica (2014): «Senza angosce. Riletture del "perturbante" freudiano», disponibile in <www.xoomer.virgilio.it/raccontarsi/interventi/farnetti_perturbante.pdf> [29/06/2014].

Foucault, Michel (1970): Le parole e le cose, Rizzoli, Milano.

FREUd, Sigmund (2006): «Il perturbante», in Totem e tabù e altri saggi di antropologia, Newton Compton, Roma. 
Fusillo, Massimo (2004-2007): «Metamorphosis at the window (Stevenson, Kafka, Cronenberg)», in Elephant \& Castle, n. 0, disponibile in <www.unibg.it/cav/elephantandcastle> [29/06/2014].

GuAltieri, Mariangela (2004): «Paesaggio con fratello rotto», disponibile in <http:// www.teatrovaldoca.org/pdf/schede_teatrografia_valdoca_pdf/paesaggio_ con_fratello_rotto_scheda_sito.pdf> [29/06/2014].

GuARINO, Raimondo (2001): «L'apocalisse teatrale della Socìetas Raffaello Sanzio», disponibile in <http://www-static.cc.univaq.it/culturateatrale/materiali/Guarino/guarino-indi.php> [29/06/2014].

Hillaert, Wouter e Crombez, Thomas (2005): «Cruelty in the Theatre of the Societas Raffaello Sanzio», intervento al convegno Tragedy, the Tragic, and the Political, tenutosi il 24 marzo 2005 a Leuven, disponibile in <http://homepages.ulb.ac. be/ rgeerts/inlthewet/castelluci.pdf $>$ [29/06/2014].

JACKsON, Rosemary (1986): Il fantastico. La letteratura della trasgressione, Tullio Pironti, Napoli.

Jung, Carl Gustav (2004): L'io e l'inconscio, Bollati Boringhieri, Torino.

Lehmann, Hans-Thies (1999): Postdramatisches Theater, Verlag der Autoren, Frankfurt am Main.

MANGo, Lorenzo (2010): «Il problema del dramma nell'epoca del postdrammatico», Prove di drammaturgia, vol. XVI, n. 1, pp. 39-44.

(2003): La scrittura scenica. Un codice e le sue pratiche nel teatro del Novecento, Bulzoni, Roma.

MANIERI, Flavio (2006): Psicoanalisi e antropologia nella genesi dello studio freudiano sulla cultura, (1968) prefazione a Freud, Totem e tabù, Newton Compton, Roma, pp. 8-45.

MANZeLla, Gianni (2014): «Kaddish per quattro creazioni della Socìetas Raffaello Sanzio", in Doppiozero, disponibile in <http://www.doppiozero.com/materiali/speciali/lettere-romeo-castellucci-io-ricordo> [29/06/2014].

Pasqualicchio, Nicola (2013a): La meraviglia e la paura. Il fantastico nel teatro europeo (1750-1950), Bulzoni, Roma.

(2013b): «Case di morti. L'interno domestico come spazio perturbante tra il teatro antico e la drammaturgia di Maeterlinck e Strindberg», in Brumal, vol. I, n. 1, pp. 79-101, disponibile in <http://revistes.uab.cat/brumal/issue/view/v1-n12013> [30/06/2014].

Ponte Di Pino, Oliviero (2006): «Il segno e il sangue: Paesaggio con fratello rotto. Una nota sulla trilogia del Teatro Valdoca», ateatro. webzine di cultura teatrale, n. 104, disponibile in <http://www.ateatro.it/webzine/2006/10/15/il-segno-e-il-sangue-paesaggio-con-fratello-rotto $>$ [29/06/2014].

(2003): «Tragedia Endogonidia R.\#7. Il ciclo della Socìetas Raffaello Sanzio fa tappa a Roma», ateatro. webzine di cultura teatrale, n. 060, disponibile in <http:// www.ateatro.it/webzine/?s=tragedia\%20endogonidia\%20R\#07> [29/06/2014].

RABKIN, Eric S. (2007): «Metamorphosis, the Mechanisms of Repression, and the Evolution of the Unconscious in European Literature», in RILUNE, n. 6, pp. 45-60.

RANK, Otto (2001): Il doppio. Uno studio psicoanalitico, Se SrL, Milano. 
Ridout, Nicholas (2006): «Make-believe. Socìetas raffaelo Sanzio do theatre», in Joe Kelleher, Nicholas Ridout (ed.), Contemporary Theatres in Europe. A critical companion, Routledge, London and New York. $<$ http://dx.doi.org/10.4324/9780203391297_chapter_12 >

Rimond, Giorgio (2001): «Il fantastico come sintomo", in Accademia Roveretiana degli Agiati. Atti, VIII serie, vol. I, pp. 285-297.

SÁnchez-BioscA, Vicente (1999): «Metamorphosis as Fantasy of the Hybrid», in Rita de Grandis, Zilà Bernd (ed.), Unforeseable Americas: Questioning Cultural Hybridity in the Americas, Critical Studies, n. 13, Amsterdam/Atlanta, pp. 283-300.

Socìttas Raffaello Sanzio (2007): Tragedia Endogonidia, Raro Video, Eccentriche Visioni.

Throop, Jason C. (2005): «Hypocognition, a "Sense of the Uncanny", and the Anthropology of Ambiguity: Reflections on Robert I. Levy's Contribution to Theories of Experience in Anthropology», ETHOS, vol. 33, n. 4, pp. 499-511.

Todorov, Tzvetan (2007 [1970]): La letteratura fantastica, Garzanti, Milano.

Vella, Alexei (2011): It Lacks A Human Corpse, a Thesis Exhibition and Suporting Paper presented to the Ontario College of Art and Design University, Canada, disponibile in <http://www.alexeivella.com/wp-content/uploads/2014/01/vella_alexei_ thesis_ilahc_site.pdf $>$ [30/06/2014].

Vergine, Lea e Verzotti, Giorgio (2004): Il Bello e le bestie. Metamorfosi, artifici e ibridi dal mito all'immaginario scientifico, SKIRA, Milano.

ZANGRANDi, Silvia (2011): Cose dell'altro mondo. Percorsi nella letteratura fantastica italiana del Novecento, ArchetipoLibri, Bologna. 